\title{
Age - Group Differences in Reactive Strength and Measures of Intra-Day Reliability in Female International Footballers
}

\author{
Brian Doyle1, Declan Browne1, Dan Horan².
}

\section{'Department of Science and Health, Institute of Technology, Carlow, ${ }^{2}$ High Performance Department, Football} Association of Ireland, Abbottstown, Dublin.

\section{ABSTRACT}

The aim of this present study was to compare the reactive strength index (RSI) characteristics and trial-to-trial reliability of U17, U19, and Senior female international soccer players. Fifty - seven elite female soccer players participated in the study, (age: 18.1 + 3.1 years; height: $167.5+6.3 \mathrm{~cm}$; weight: $61.84+$ $7.7 \mathrm{~kg}$ ). Participants performed 3 maximal repetitions of the 10/5 repeated jump test (10/5RJT) following a specific warm-up and familiarisation protocol. Senior players possessed higher levels of RSI with a large effect size present when compared with the U17 $(\mathrm{P}=0.043, \mathrm{ES}=.97)$ and U19 $(\mathrm{ES}=0.85)$ age groups. Trivial differences $(E S=0.17)$ in $R S I$ existed between the U17 and U19 age categories. Trial-to-trial analyses demonstrated 10/5 RJT RSI to possess adequate levels of reliability with a range of mean coefficients of variance (CV) of $3.1-7.3$ $\%$ and intraclass correlation (ICC) between 0.95 0.98 present across all three age groups. However, large variations in the between-athlete $\mathrm{CV}$ for $\mathrm{RSI}$ were revealed ranging from $1-27 \%, 0.4-10.3 \%$, and $1-7 \%$ for U17, U19, and senior age groups respectively. These results suggest that age groups can distinguish reactive strength capabilities. In addition, female international footballers with higher levels of RSI appear to produce more reliable measures of RSI via the 10/5 RJT.

Keywords: Monitoring, Female athletes, Vertical jump, SSC, Soccer

\section{INTRODUCTION}

International level football players participate in numerous periods of fixture congestion whereby players are required to play in two or more games per week on numerous occasions throughout a season (1). Due to increased competition schedules, training demands, and travel full recovery postmatch play may not always be possible (2). Therefore, balancing sufficient recovery, from the training load sustained at club level in combination with applying the necessary training load exposures to ensure international competition readiness can present a complex scenario (3).

Football is an intermittent field sport where players transition from long periods of aerobic activity to short bouts of high-intensity anaerobic activities such as high-speed running and sprinting (2). The physical demands of the game have been seen to elicit both acute and residual forms of fatigue within and post-match play (4). In addition, football specific actions such as shooting, passing, tackling, and jumping are executed in tandem with accelerations, decelerations, and changes of direction at varying speeds. Such actions have been associated with increased levels of muscle damage, leading to a decline in muscular force production which may potentially inhibit physical performance in the subsequent days (5). Therefore, coaches and sports science staff are now employing a more scientific approach to the monitoring of their player's training programs and competition schedules with the aim of limiting fatigue, maximising recovery, and maintaining competition readiness $(1,6)$.

However, unless the chosen monitoring test protocol and measurement tool are known to be reliable, valid, and sensitive any attempt to effectively observe potential changes in neuromuscular status may be deemed ineffective (1). To establish reliability, the criteria of a coefficient of variance 
$(\mathrm{CV})<10 \%$ and intraclass correlation $(\mathrm{ICC})>.8$ must be obtained (7). Then to maximise the impact of any monitoring data coaches and sports science staff need to optimise the data interpretation process. Coaches need to be able to differentiate between the test noise coefficient of variance $(\mathrm{CV})$ and the smallest worthwhile change (SWC) in test scores. The SWC is the smallest change in athletic performance that is meaningful (8). Should a test lack the capacity to identify the smallest meaningful changes in performance in response to a training or performance stimulus, the ability to effectively determine a true change in neuromuscular status is severely hindered (8). When an assessment of neuromuscular status possesses the required levels of sensitivity to detect true meaningful changes in performance, coaches, sports scientists, and medical teams can make more informed decisions regards player management and training load prescription in the presence of neuromuscular fatigue (1).

An extensive body of work exists within the literature demonstrating CMJ to be the most utilised jump protocol for the assessment of neuromuscular fatigue $(5,9)$. The $\mathrm{CMJ}$ is classed as a reactive strength movement, where force production is predominately driven by musculotendinous structures and the SSC (10). Reactive strength has been defined as the ability to switch quickly from an eccentric to concentric contraction (11). Greater reactive strength capabilities have been related to enhanced $10 \mathrm{~m}$ acceleration and change of direction ability, both important movement qualities in football performance $(12,13)$. Furthermore, reactive strength movements can be classed as possessing either slow or fast SSC features (14). Slow SSC movements (ground contact times $>250$ milliseconds) such as the CMJ exhibit longer contraction times and greater displacements of the hip, knee, and ankle while fast SSC movements (ground contact times $<250$ milliseconds) display shorter contraction times and smaller displacements of the hip, knee, and ankle $(15,16)$. Yet the sensitivity of countermovement jump height has been questioned within the literature as players tend to make slight adjustments to their jump technique to obtain a specific height which may hide evidence of neuromuscular fatigue (17). Several researchers have revealed variables such as reactive strength index (RSI) and flight time contraction time ratio demonstrates a greater ability to detect changes in neuromuscular status $(10,18)$.

The drop jump protocol is another form of fast SSC movement assessment and is frequently used to measure RSI capabilities in both elite male (16) and female athlete populations (19). Only a number of studies have revealed RSI values for female athletes in handball, rugby, basketball, and football via various drop jump tests (19-22), with only Emmonds et al. 2019 reporting intra-day reliability values (CV $=3.5 \%, I C C=0.93$ ) for $40 \mathrm{~cm}$ drop jump RSI (19). Due to the technical aspects of performing the drop jump, a familiarisation period may be required for players, as well as the identification of each individual player's optimal drop height. Therefore, it may not be the most appropriate RSI assessment method in an applied setting $(15,23,24)$. As the feasibility for both those processes to take place within an international football training camp may not be possible due to limited access to players due to training, competition, and travel schedules.

The 10/5 repeated jump test (10/5 RJT) is a nonevasive and non-fatiguing method of assessing an individual's fast SSC abilities (25). Currently, limited data exists detailing the reliability of reactive strength index (RSI) via 10/5 RJT in female team sport athletes. Only one study has assessed the reliability of the 10/5 RJT measurement of RSI (26). Coymns et al (2019) reported interday reliability of $(C V=10 \%$, ICC $=0.91$ ) in female collegiate team sport (26). While the reliability and validity of several jump tests have been assessed in elite male populations the topic has been poorly investigated in elite female populations $(10,16,18,25)$. Considering there are innate physiological and match demand differences between the sexes, specific analysis of elite female athletes is needed (27 -30). Furthermore, the sensitivity of any neuromuscular status assessment is paramount but the knowledge available on the sensitivity of jump tests in elite female football players is scarce $(31,32)$. Therefore, the purpose of this study was to compare the performance characteristics of 10/5 RJT RSI and trial-to-trial reliability of $\mathrm{RSI}$ and associated variables contact time (CT) and jump height (JH) in U17, U19, and senior female international football players.

\section{METHODS}

\section{Experimental Approach to Problem}

This was a cross-sectional comparative study aimed at describing the trial-to-trial and performance characteristics of RSI derived from the 10/5 repeated jump test (10/5 RJT) in U17, U19, and senior female international football players. The 10/5 RJT assessments were conducted as part of each team's performance testing program. 
Testing sessions took place in the afternoon (between 12:00 and 16:00) on the first day of each team's respective training camp during 2019.

\section{Subjects}

Fifty-seven female international football players selected to represent the $\mathrm{U} 17$ ( $\mathrm{n}=24$; age: 16.5 + 0.4 years; height: $167.5+6.3 \mathrm{~cm}$; weight: 61.84 + $7.7 \mathrm{~kg}$ ), $\mathrm{U} 19$ ( $\mathrm{n}=23$; age: 17.8 + 0.6 years; height: $166.7+6.1 \mathrm{~cm}$; weight: $61.5+7.6 \mathrm{~kg}$ ) or senior ( $n=10$; age: $22.7+5.2$ years; height: 170 $+6.3 \mathrm{~cm}$; weight: $67.5+7.8 \mathrm{~kg}$ ) international teams volunteered to participate in the study. Ethical approval was obtained by the College Research Ethics Committee. The participants were informed of the risks of the study in person and in writing and signed an informed consent document before the beginning of data collection and were free to withdraw from the study at any time. For those under the age of 18 years age parental or guardian signed consent was obtained. The study was conducted in accordance with the Declaration of Helsinki.

\section{Procedures}

All players took part in one testing session where three maximum effort trials of the 10/5 RJT were completed. The 10/5 RJT has been deemed a reliable and valid assessment method of RSI in female team sport athletes (26). The 10/5 RJT required the participants to perform a counter movement jump followed by 10 maximal rebound jumps (25). Each participant underwent a familiarisation trial of the 10/5 RJT, whereby the participant had the test protocol demonstrated to them by a researcher before performing a single submaximal effort. Prior to the initiation of the testing session, participants underwent a structured 10 minute warm-up protocol consisting of jogging, running, lower limb mobility, and dynamic stretching movements followed by countermovement jump (CMJ) (2 repetitions) and CMJ into pogo hops (2 sets $\times 10$ hops) $(15,33)$. On completion of the warm-up, a 5-minute recovery period was employed to assuage any effects of fatigue. Participants then completed 3 trials of the 10/5 RJT with a minimum of one minute's rest between each trial (25). During the test, participants were instructed to maintain hands akimbo to standardise the protocol and negate upper body interference (34). Additionally, participants were asked to maximise jump height and minimise ground contact time, specifically prompted "imagine the floor was a hot surface, jump as high as possible and to imagine their leg is like a stiff spring rebounding off the ground" (35). Any participants deemed unfit by the medical staff were omitted from the study.

The Optojump (Microgate, Bolzano, Italy) photocell system was used to measure each 10/5 RJT trial. The Optojump (Microgate, Bolzano, Italy) system recorded CT (s) and FT (s) and automatically derived $\mathrm{JH}(\mathrm{m})$ from the flight time using the equation $\mathrm{JH}(\mathrm{m})=($ gravity $X$ flight time) $2 / 8$, where gravity $=9.81 \mathrm{~m} . \mathrm{s}-1$ (36). RSI is then calculated by utilising the acquired data in the following equation $\mathrm{RSI}=\mathrm{JH}(\mathrm{m}) / \mathrm{CT}(\mathrm{s})$ (37). RSI was calculated for each jump utilising the aforementioned equations, the 5 best non-consecutive jump heights with a ground contact time of less than $250 \mathrm{~ms}$ were averaged to define an overall RSI for each trial (25).

\section{Statistical Analysis}

Means and standard deviations (SD) were calculated for 10/5 RJT variables RSI, contact time (CT) jump height $(\mathrm{JH})$ for all age groups. The assumption for normality was confirmed using the Shapiro-Wilks test. A one-way analysis of variance was used to investigate the differences in 10/5 RJT variables between the age groups. When the $F$ test was significant $(P<0.05)$, Bonferroni post-hoc pairwise comparisons were performed to identify the level of difference between age groups with SPSS, version 25.0, SPSS Inc, Chicago, IL, USA. To determine the magnitude of difference between the age groups, the effect size (ES: Cohens d) was calculated, and values of 0.2,0.5 and above 0.8 were interpreted as small, medium, and large, respectively (38). The trial-to-trial (Jump $1 \mathrm{v}$ jump $2 \vee$ jump 3 ) reliability of each 10/5 RJT variable was calculated. Reliability was assessed using the coefficient of variation (CV) using Microsoft excel spreadsheet (39) and intraclass correlation (ICC) with 95\% confidence intervals with SPSS, version 25.0, SPSS Inc, Chicago, IL, USA. Acceptable reliability was determined when an ICC $>0.8$ and a CV $<10 \%$ were both met ( 7$)$. The $\mathrm{CV}$ was used to provide an indication of the error value or noise within the test $(\mathrm{CV} \%=$ within-subject SD/ mean * 100) (8). McGuigan (2014) highlighted that the SWC characterises the smallest change that is of benefit to athletic performance (8). The SWC was calculated by multiplying the between-subject SD by 0.2 , which represents a small effect. If the CV was < SWC the related variable would be deemed capable of detecting the SWC (16). Individual trialto-trial CV was calculated to highlight the betweensubject variability of reliability within a team setting. 


\section{Results}

Table 1 displays the results of 10/5 RJT RSI, $\mathrm{CT}$, and $\mathrm{JH}$ across all age groups. Age group influenced RSI performance with senior players demonstrating greater RSI scores than the U17 (ES $=0.97)$ and $U 19(E S=0.85)$ age groups whilst trivial differences ( $E S=0.17$ ) existed between the U17 and U19 players (Table 1). U17 players displayed shorter CT when compared to U19 (ES = 0.79) and senior ( $E S=0.17$ ) age groups. Senior players presented higher $\mathrm{JH}$ scores as moderate (ES = $0.55)$ to large ( $E S=1.27$ ) effect sizes were present when compared to the U19 and U17 age groups respectively. A moderate effect size $(E S=0.58)$ existed between U17 and U19 JH scores (Table 1).
Table. 2 contains mean + SD, coefficient of variation (CV), intraclass correlation (ICC), confidence intervals $(\mathrm{Cl})$, and smallest worthwhile change (SWC) for the 10/5 RJT variables RSI, CT, and JH across all age groups. Individual player trial-to-trial CV range for all 10/5 RJT variables is shown for each age group in figure 1 . The 10/5 RJT variables were only reliable if the criteria of a CV $<10 \%$ and ICC $>0.80$ were established. All 10/5 RJT variables RSI, CT, and $\mathrm{JH}$ demonstrated acceptable levels of reliability versus criteria across all three age groups. However, all 10/5 RJT variables across each age group were unable to detect the SWC due to revealed greater CV values than the calculated SWC (Table 2).

Table.1 Age group descriptive statistics (mean + SD) of 10/5 RJT RSI, CT, and JH

\begin{tabular}{|c|c|c|c|c|}
\hline Participants & No. & $\begin{array}{c}\text { RSI } \\
(\mathbf{m} / \mathbf{s})\end{array}$ & $\begin{array}{c}\text { Contact Time } \\
\text { (sec) }\end{array}$ & $\begin{array}{c}\text { Jump Height } \\
\text { (cm) }\end{array}$ \\
\hline Senior & 10 & $1.57 \pm .24^{*}$ & $.180 \pm .017$ & $28.24 \pm 3.45^{*}$ \\
\hline U19 & 23 & $1.35 \pm .28$ & $.192 \pm .013^{\star}$ & $25.83 \pm 5.20$ \\
\hline U17 & 24 & $1.30 \pm .31$ & $.178 \pm 0.22$ & $22.90 \pm 4.86$ \\
\hline
\end{tabular}

${ }^{*}$ denotes significantly $(P=<0.05)$ different to $U 17$
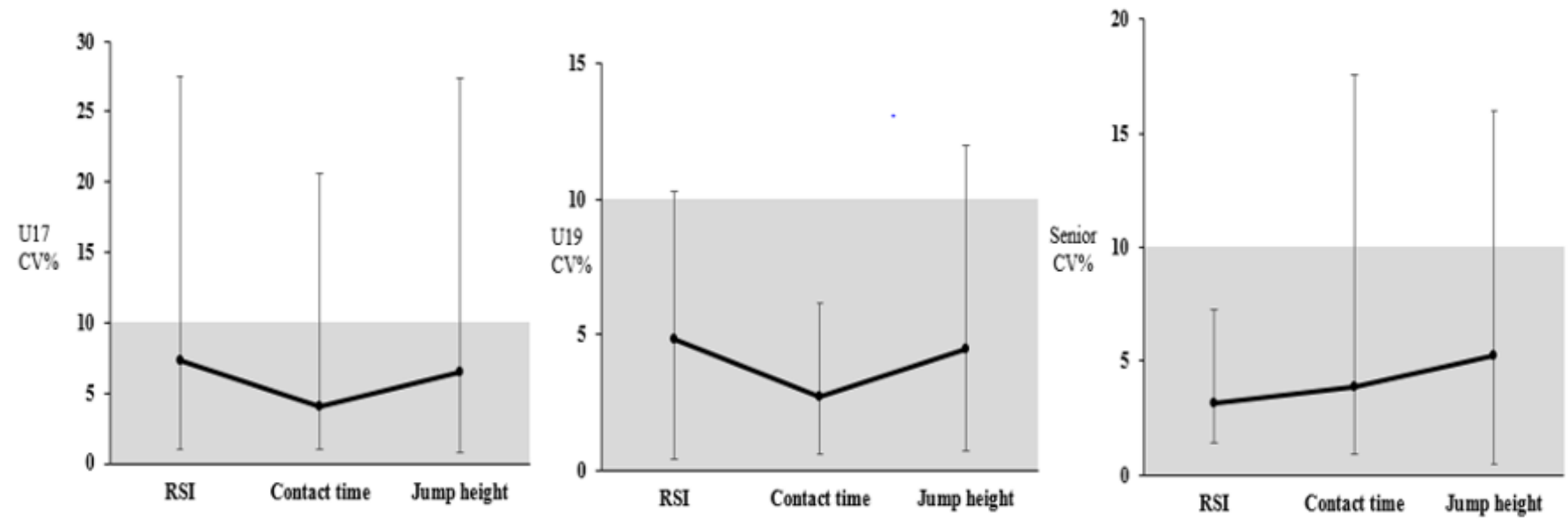

Figure 1. Between athlete CV range for 10/5 RJT variables RSI, CT and JH in U17, U19 and senior age groups. Gray shaded area $=$ zone of acceptable reliability $($ CV\% $<10 \%)$. 
Table 2. Age group 10/5 RJT Trial-to-Trial Reliability Variables

\begin{tabular}{|c|c|c|c|c|c|c|c|}
\hline \multirow{2}{*}{ Age Group } & \multirow[b]{2}{*}{ Variables } & \multirow[b]{2}{*}{ CV (\%) } & \multirow[b]{2}{*}{ ICC } & \multicolumn{2}{|c|}{$95 \% \mathrm{Cl}$} & \multirow[b]{2}{*}{ SWC (\%) } & \multirow[b]{2}{*}{$\mathrm{CV}<\mathrm{SWC}$} \\
\hline & & & & Lower & Higher & & \\
\hline \multirow{3}{*}{ Seniors } & $\mathrm{RSI}(\mathrm{m} / \mathrm{s})$ & 3.15 & 0.98 & 0.96 & 0.99 & 3.03 & No \\
\hline & $\begin{array}{l}\text { Contact } \\
\text { time (sec) }\end{array}$ & 3.90 & 0.86 & 0.61 & 0.96 & 1.87 & No \\
\hline & $\begin{array}{c}\text { Jump } \\
\text { Height } \\
\text { (cm) }\end{array}$ & 5.22 & 0.92 & 0.76 & 0.98 & 2.44 & No \\
\hline \multirow{3}{*}{ U19 } & $\mathrm{RSI}(\mathrm{m} / \mathrm{s})$ & 4.95 & 0.98 & 0.95 & 0.99 & 4.13 & No \\
\hline & $\begin{array}{l}\text { Contact } \\
\text { time (sec) }\end{array}$ & 2.75 & 0.91 & 0.82 & 0.96 & 1.34 & No \\
\hline & $\begin{array}{c}\text { Jump } \\
\text { Height } \\
(\mathrm{cm})\end{array}$ & 4.49 & 0.98 & 0.96 & 0.99 & 4.03 & No \\
\hline \multirow{3}{*}{ U17 } & $\mathrm{RSI}(\mathrm{m} / \mathrm{s})$ & 7.28 & 0.95 & 0.89 & 0.97 & 4.82 & No \\
\hline & $\begin{array}{c}\text { Contact } \\
\text { time (sec) }\end{array}$ & 3.98 & 0.94 & 0.88 & 0.97 & 2.55 & No \\
\hline & $\begin{array}{c}\text { Jump } \\
\text { Height } \\
\text { (cm) }\end{array}$ & 6.50 & 0.97 & 0.93 & 0.98 & 3.44 & No \\
\hline
\end{tabular}

\section{DISCUSSION}

The aim of this study was to compare the 10/5 RJT RSI characteristics and trial-to-trial reliability of 10/5 RJT RSI and associated variables (CT and $\mathrm{JH}$ ) from different age groups of female international footballers. The results of the study revealed senior players possessed superior levels of RSI primarily down to the combination of shorter CT and increased JH during the 10/5 RJT when compared to the U17 and U19 age groups (Table 1). The findings of this study mirror similar trends established within the literature with senior international players demonstrating greater vertical jump performances (i.e. countermovement and squat jump) than their younger counterparts (40 42). It has been previously debated that biological maturation in females ceases at 17 years of age and any improvements in physical performance tests in older age groups may be attributed to physiological adaptations elicited by exposure to higher total training loads and an increase in match demands (40). Considering, senior players have been seen to experience higher volume and intensities of running demands during international competition than their U17 equivalents (43). It is plausible that a training program specifically designed to meet the increased match and training demands of senior female professional football elicits physiological adaptations such as greater stretch reflex, enhanced neural potentiation, and increased elastic energy reutilisation which may enhance reactive strength capabilities $(40,44)$.

The trial-to-trial reliability of all three 10/5RJT variables of RSI, CT, and $\mathrm{JH}$ was confirmed throughout all age groups (Table.2). In terms of RSI, these findings support the work of Harper et al. (2011) and Comyns et al. (2019) regarding interday reliability of RSI measurement by the 10/5 RJT $(25,26)$. Interestingly, this study highlighted increased 10/5 RJT RSI test scores were associated with greater levels of reliability. Seniors players reported mean RSI scores of $1.57+.24 \mathrm{~m} / \mathrm{s}$ and an RSI of (CV 3.15\%) whilst less accurate levels of 10/5 RJT RSI reliability were evident in U17 (CV 7.28\%) and U19 (CV 4.95\%) cohorts with significantly lower RSI scores of 1.30 $+.31 \mathrm{~m} / \mathrm{s}$ and $1.35+.28 \mathrm{~m} / \mathrm{s}$. Additionally, the trialto-trial reliability of CT and $\mathrm{JH}$ via the 10/5 RJT has yet to be reported within the literature (Table 2). The intra-day reliability of $\mathrm{JH}(\mathrm{CV}=3.20 \%, \mathrm{ICC}=$ $0.93)$, and $\mathrm{RSI}(\mathrm{CV}=3.50 \%, \mathrm{ICC}=0.93)$ has been reported in female club level footballers (19) and CT $(\mathrm{CV}=8.40 \%, \mathrm{ICC}=0.85)$ and $\mathrm{JH}(\mathrm{CV}=5.73 \%, \mathrm{ICC}$ $=0.70$ ) in male youth rugby players (16) with both cohorts implementing a $40 \mathrm{~cm}$ drop jump protocol. Additionally, Cormack et al. (2008) reported a CV of $5.2 \%$ for $\mathrm{JH}$ in a counter movement jump in male AFL players (10). 
In establishing the test trial-to-trial reliability, a comparison between the test error value or noise $(\mathrm{CV})$ and the change in performance of a designated variable can aid coaches and sports scientists to determine a truly meaningful change in neuromuscular status (16). The SWC is the smallest change in a variable that is of benefit to athletic performance (8). For example, should the change in a players' RSI be greater than the CV of the test it may be deemed a worthwhile increase or decrease in RSI (45). This process may lead to improved data interpretation potentially affording coaches and sports scientists the opportunity to make more informed decisions regards training load prescription to optimise player readiness.

The trial-to-trial SWC for 10/5 RJT RSI for each age group was 3.03\% (seniors), 4.13\% (U19), and $4.82 \%$ for (U17). However, their respective mean RSI CV values for the seniors were (3.15\%), U19 (4.95\%), and U17 (7.28\%) which was greater than their relevant SWC indicating a lack of capacity to identify the SWC in RSI for any age group. These results add further support to the conclusion of Comyns et al. (2019) that the 10/5 RJT was not capable of identifying the SWC in RSI in female team sport populations (26). RSI is derived by dividing $\mathrm{JH}$ by CT and the RSI value may be influenced by a decrease or increase in $\mathrm{JH}$ or the same in CT (35). One may suggest that the interaction between $\mathrm{JH}$ and CT potentially inhibits the 10/5 RJT ability to detect the SWC change in RSI as it is dependent on the dynamic relationship between two variables.

Similar, to Beattie and Flanagan (2015) all 10/5 RJT variables revealed a certain degree of noise from an individual player aspect in U17, U19, and senior age groups (16). Although, RSI, CT, and JH, were deemed to be reliable measures in all three age groups (Table 2), considerable individual differences in the variance of performance existed between the players in each group (Figure 1). The between athlete RSI CV ranged from (1.4 - $7.2 \%)$, $(0.4-10.3 \%)$ and $(1.1-27.5 \%)$ for senior, U19, and $U 17$ age groups respectively, indicating that the measurement of RSI via the 10/5 RJT to be less reliable in the $\mathrm{U} 17$ cohort. Further emphasising players possessing higher reactive strength levels provide more accurate measures of reliability. On the basis of this evidence, reliability can differ from player to player and age group to age group. By using the group mean CV \% to detect changes in neuromuscular status the test may lack the required levels of sensitivity to identify a meaningful change in some individuals (16). This study is reflective of a small sample size of elite female international footballers therefore the generalisation of these results should be interpreted with caution. Future work should also look to investigate the interday and intra-day reliability and response of all 10/5 RJT variables, across various age groups in both male and female cohorts of elite team sport athletes. As the responses may be decidedly different from those present in this study due to numerous influencing factors such as gender, training age, and competition level.

\section{PRACTICAL APPLICATIONS}

The findings of this study provide age-appropriate data for coaches, strength and conditioning, and sports scientists working with elite female international players at multiple age groups. The ability to assess the physical performance characteristics of female football players and establish cohort-specific levels of reliability can aid development pathways in the individualisation and monitoring of training programs. The study revealed that RSI could be differentiated by age group. Furthermore, the data indicated that players with low levels of reactive strength are associated with less reliable measurements of RSI. As a result, measurement of RSI via the 10/5 RJT may not be an effective measurement method of changes in neuromuscular status in populations displaying low levels of RSI. All variables measured by the 10/5 RJT were reliable measures from trial-totrial. However, RSI, CT, and JH were unable to detect the SWC in elite female international footballers. Additionally, when monitoring any playing group, a high degree of variation of reliability will be present between players, therefore each individual player's CV should be utilised to establish true meaningful change on an individual basis, allowing for a more informed and individualised approach to player management (46).

\section{ACKNOWLEDGMENTS}

We would like to thank the players and staff at the Football Association of Ireland for their hard work and cooperation throughout this study.

\section{DISCLOSURE STATEMENT}

No potential conflict of interest was reported by the authors. 


\section{FUNDING}

Funding was provided by the president's fellowship research award from the Institute of Technology, Carlow, and the Football Association of Irelands, high-performance department.

\section{REFERENCES}

1. Thorpe, R.T., Atkinson, G., Drust, B. and Gregson, W., 2017. Monitoring fatigue status in elite team-sport athletes: implications for practice. International journal of sports physiology and performance, 12(s2), pp. S2-27. https://journals.humankinetics.com/view/ journals/ijspp/12/s2/article-pS2-27.xml

2. Datson, N., Hulton, A., Andersson, H., Lewis, T., Weston, M., Drust, B. and Gregson, W., 2014. Applied physiology of female soccer: an update. Sports Medicine, 44(9), pp.1225-1240. https://research. tees.ac.uk/ws/files/6311830/317310.pdf

3. Buchheit, M. and Dupont, G., 2018. Elite clubs and national teams: sharing the same party?https://www. tandfonline.com/doi/full/10.1080/24733938.2018.147 0156

4. Hader, K., Rumpf, M.C., Hertzog, M., Kilduff, L.P., Girard, O. and Silva, J.R., 2019. Monitoring the athlete match response: Can external load variables predict post-match acute and residual fatigue in soccer? A systematic review with meta-analysis. Sports Medicine-Open, 5(1), p.48. https://link.springer.com/article/10.1186/s40798-019-0219-7

5. Nédélec, M., McCall, A., Carling, C., Legall, F., Berthoin, S. and Dupont, G., 2012. Recovery in soccer. Sports medicine, 42(12), pp.997-1015.https://hal-insep.archives-ouvertes.fr/hal-01660950/document

6. Halson, S.L., 2014. Monitoring training load to understand fatigue in athletes. Sports medicine, 44(2).

7. Hopkins, W.G., 2000. Measures of reliability in sports medicine and science. Sports medicine, 30(1), pp.1-15. https://link.springer.com/article/10.2165/00007256-200030010-00001

8. McGuigan, M., 2014. Evaluating athletic capacities. High-performance training for sports, pp.3-15

9. Silva, J.R., Rumpf, M.C., Hertzog, M., Castagna, C., Farooq, A., Girard, O. and Hader, K., 2018. Acute and residual soccer match-related fatigue: a systematic review and meta-analysis. Sports Medicine, 48(3), pp.539-583.https://link.springer.com/article/10.1007/ s40279-017-0798-8

10. Cormack, S.J., Newton, R.U. and McGuigan, M.R., 2008. Neuromuscular and endocrine responses of elite players to an Australian rules football match. International journal of sports physiology and performance, 3(3), pp.359-374.https://ro.ecu.edu.au/cgi/ viewcontent.cgi?article $=1019 \&$ context $=$ theses

11. Young, W., 1995. Laboratory strength assessment of athletes. New studies in athletics, 10, pp.89-89.

12. Lockie, R.G., Murphy, A.J., Knight, T.J. and de Jonge,
X.A.J., 2011. Factors that differentiate acceleration ability in field sport athletes. The Journal of Strength \& Conditioning Research, 25(10), pp.2704-2714.https:// journals.Iww.com/nsca-jscr/FullText/2011/10000/Factors_That_Differentiate_Acceleration_Ability_in.9.as$\mathrm{px}$

13. Young, W.B., James, R. and Montgomery, I., 2002. Is muscle power related to running speed with changes of direction?. Journal of sports medicine and physical fitness, 42(3), pp.282-288. https://www.researchgate. net/profile/Warren_Young/publication/11281917_ Is_Muscle_Power_Related_to_Running_Speed_ With_Changes_of_Direction/links/Odeec529cfa284fa7d000000.pdf

14. Komi, P.V., 2000. Stretch-shortening cycle: a powerful model to study normal and fatigued muscle. Journal of biomechanics, 33(10), pp.1197-1206. http://citeseerx.ist.psu.edu/viewdoc/download?$\mathrm{doi}=10 \cdot 1 \cdot 1 \cdot 915 \cdot 5087 \&$ rep $=$ rep1\&type $=p d f$

15. Markwick, W.J., Bird, S.P., Tufano, J.J., Seitz, L.B. and Haff, G.G., 2015. The intraday reliability of the reactive strength index calculated from a drop jump in professional men's basketball. International journal of sports physiology and performance, 10(4), pp.482488.

16. Beattie, K. and Flanagan, E.P., 2015. Establishing the reliability \& meaningful change of the dropjump reactive strength index. J Aust Strength Cond, 23(5), pp.12-18.https://www.researchgate. net/profile/Kris_Beattie/publication/283479348_Establishing_the_Reliability_Meaningful_Change_ of_the_Drop-Jump_Reactive-Strength_Index/ links/5639bb3308ae4624b75f1325/Establishing-the-Reliability-Meaningful-Change-of-the-Drop-Jump-Reactive-Strength-Index.pdf

17. Balloch, A.S. (2018), Evaluation of Kinetic and kinematic variables during a countermovement jump to assess neuromuscular status in team-sport athletes ', Journal of Australian Strength and Conditioning, 26(1), pp. 49-56.

18. Oliver, J.L., Lloyd, R.S. and Whitney, A., 2015. Monitoring of in-season neuromuscular and perceptual fatigue in youth rugby players. European Journal of Sport Science, 15(6), pp.514-522.https://www. researchgate.net/profile/Rhodri_Lloyd3/publication/281779082_Monitoring_of_inseason_neuromuscular_and_perceptual_fatigue_in_youth_rugby_players/links/56a2068d08ae24f627045f1c/ Monitoring-of-in-season-neuromuscular-and-perceptual-fatigue-in-youth-rugby-players.pdf

19. Emmonds, S., Nicholson, G., Begg, C., Jones, B. and Bissas, A., 2019. Importance of physical qualities for speed and change of direction ability in elite female soccer players. The Journal of Strength \& Conditioning Research, 33(6), pp.1669-1677.prints.leedsbeckett.ac.uk/id/eprint/3934/1/Emmonds\%20et\%20 al. \%20IMPORTANCE\%200F\%20PHYSICAL\%20 QUALITIES\%20FOR\%20SPEED\%20AND\%20 CHANGE\%200F\%20DIRECTION\%20ABILITY\%20 IN\%20ELITE\%20FEMALE\%20SOCCER\%2OPLAY- 
ERS..pdf

20. Prieske, O., Chaabene, H., Puta, C., Behm, D.G., Büsch, D. and Granacher, U., 2019. Effects of drop height on jump performance in male and female elite adolescent handball players. International journal of sports physiology and performance, 14(5), pp.674680.

21. Werstein, K.M. and Lund, R.J., 2012. The effects of two stretching protocols on the reactive strength index in female soccer and rugby players. The Journal of Strength \& Conditioning Research, 26(6), pp.1564-1567.https://journals.Iww.com/nsca-jscr/ Fulltext/2012/06000/The_Effects_of_Two_Stretching_ Protocols_on_the.15.aspx

22. Zody, A., Rossi, S., McMillan, J. and Buckley, T., 2011. Under recovery and drop jump characteristics during a collegiate basketball season in female basketball players. The Journal of Strength \& Conditioning Research, 25, pp.S106-S107. https://journals. Iww.com/nscajscr/Abstract/2011/03001/Under_Recovery_and_Drop_Jump_Characteristics.168.aspx

23. Byrne, D.J., Browne, D.T., Byrne, P.J. and Richardson, N., 2017. Interday reliability of the reactive strength index and optimal drop height. Journal of strength and conditioning research, 31(3), pp.721-726.https:// www.researchgate.net/profile/Declan_Browne2/ publication/304809850_The_InterDay_Reliability_of_Reactive_Strength_Index_and_Optimal_Drop_ Height/links/5ca658b0299bf118c4b32086/ The-Inter-Day-Reliability-of-Reactive-Strength-Index-and-Optimal-Drop-Height.pdf

24. Byrne, P.J., Moody, J.A., Cooper, S.M. and Kinsella, S., 2017. The reliability of countermovement jump performance and the reactive strength index in identifying drop-jump drop height in hurling players.

25. Harper, D., Hobbs, S. and Moore, J., 2011. The ten to five repeated jump test: A new test for evaluation of lower body reactive strength. In British Association of Sports and Exercises Sciences Student Conference. BASES: Chester, United Kingdom.

26. Comyns, T.M., Flanagan, E.P., Fleming, S., Fitzgerald, E. and Harper, D.J., 2019. Interday Reliability and Usefulness of a Reactive Strength Index Derived From 2 Maximal Rebound Jump Tests. International Journal of Sports Physiology and Performance, 14(9), pp.1200-1204. https://ray.yorksj.ac.uk/id/ eprint/3770/1/IJSPP\%20TC\%20Accepted\%20Version.pdf

27. Emmonds, S., Heyward, O. and Jones, B., 2019b. The challenge of applying and undertaking research in female sport. Sports Medicine-Open, 5(1), p.51. https:// link.springer.com/article/10.1186/s40798-019-0224-X

28. Bradley, P.S., Dellal, A., Mohr, M., Castellano, J. and Wilkie, A., 2014. Gender differences in match performance characteristics of soccer players competing in the UEFA Champions League. Human movement science, 33, pp.159-171. https://www.researchgate. net/profile/DellalA/publication/258059018_Gender_ differences_in_match_performance_characteristics_ of_soccer_players_competing_in_the_UEFA_Cham-
pions_League/links/5fb68b3e299bf104cf5b9317/ Gender-differences-in-match-performance-characteristics-of-soccer-players-competing-in-the-UEFA-Champions-League.pdf

29. Baumgart, C., Hoppe, M.W. and Freiwald, J., 2014. Different endurance characteristics of female and male german soccer players. Biology of sport, 31(3), p.227. https://www.ncbi.nlm.nih.gov/pmc/articles/ PMC4135068/

30. Mujika, I., Santisteban, J., Impellizzeri, F.M. and Castagna, C., 2009. Fitness determinants of success in men's and women's football. Journal of sports sciences, 27(2), pp.107-114.

31. Pardos-Mainer, E., Casajús, J.A. and Gonzalo-Skok, O., 2019. Reliability and sensitivity of jumping, linear sprinting and change of direction ability tests in adolescent female football players. Science and Medicine in Football, 3(3), pp.183-190. https://www. researchgate.net/profile/Elena_Pardos_Mainer/ publication/329585102_Reliability_and_sensitivity_of_jumping_linear_sprinting_and_change_of_direction_ability_tests_in_adolescent_female_football_players/links/5c423c5292851c22a37fa4c3/ Reliability-and-sensitivity-of-jumping-linear-sprinting-and-change-of-direction-ability-tests-in-adolescent-female-football-players.pdf

32. Gathercole, R., Sporer, B., Stellingwerff, T. and Sleivert, G., 2015. Alternative countermovement-jump analysis to quantify acute neuromuscular fatigue. International journal of sports physiology and performance, 10(1), pp.84-92. https:// www.researchgate.net/profile/Rob_Gathercole/ publication/262939784_Alternative_CountermovementJump_Analysis_to_Quantify_Acute_Neuromuscular_Fatigue/links/55ea561508ae65b6389c5a74/ Alternative-Countermovement-Jump-Analysis-to-Quantify-Acute-Neuromuscular-Fatigue.pdf

33. Turki, O., Chaouachi, A., Drinkwater, E.J., Chtara, M., Chamari, K., Amri, M. and Behm, D.G., 2011. Ten minutes of dynamic stretching is sufficient to potentiate vertical jump performance characteristics. The Journal of Strength \& Conditioning Research, 25(9), pp.2453-2463. https://journals. Iww.com/nscajscr/fulltext/2011/09000/Ten_Minutes_of_Dynamic_Stretching_Is_Sufficient_to.14.aspx

34. Lees, A., Vanrenterghem, J. and De Clercq, D., 2004. Understanding how an arm swing enhances performance in the vertical jump. Journal of biomechanics, 37(12), pp.1929-1940.

35. Flanagan, E.P. and Comyns, T.M., 2008. The use of contact time and the reactive strength index to optimize fast stretch-shortening cycle training. Strength \& Conditioning Journal, 30(5), pp.32-38.

36. Bosco, C., Luhtanen, P. and Komi, P.V., 1983. A simple method for measurement of mechanical power in jumping. European journal of applied physiology and occupational physiology, 50(2), pp.273-282. http:// pesquisa.ufabc.edu.br/bmclab/x/VerticalJump/BoscoEJAP83jump.pdf

37. McClymont, D., 2003. Use of the reactive strength in- 
dex (RSI) as an indicator of plyometric training conditions. In Science and Football V: The proceedings of the fifth World Congress on Sports Science and Football, Lisbon, Portugal (pp. 408-16).

38. Cohen 1988 Cohen, J., 1988. Statistical Power Analysis for the Behavioural Sciences-Second Edition. 12 Lawrence Erlbaum Associates Inc. Hillsdale, New Jersey, 13.

39. Hopkins, W.G., 2017. Spreadsheets for analysis of validity and reliability. Sportscience, 21.

40. Ramos, G.P., Nakamura, F.Y., Penna, E.M., Mendes, T.T., Mahseredjian, F., Lima, A.M., Garcia, E.S., Prado, L.S. and Coimbra, C.C., 2019. Comparison of Physical Fitness and Anthropometrical Profiles Among Brazilian Female Soccer National Teams from U15 to Senior Categories. Journal of Strength and Conditioning Research.

41. Haugen, T.A., Tønnessen, E. and Seiler, S., 2012. Speed and countermovement-jump characteristics of elite female soccer players, 1995-2010. International journal of sports physiology and performance, 7(4), pp.340-349.

42. Castagna, C. and Castellini, E., 2013. Vertical jump performance in Italian male and female national team soccer players. The Journal of Strength \& Conditioning Research, 27(4), pp.1156-1161.

43. Ramos GP, Nakamura FY, Penna EM, Wilke CF, Pereira LA, Loturco I, Capelli L, Mahseredjian F, Silami-Garcia E, Coimbra CC. Activity Profiles in U17, U20, and Senior Women's Brazilian National Soccer Teams During International Competitions: Are There Meaningful Differences? The Journal of Strength \& Conditioning Research. 2019 Dec 1;33(12):3414-22.

44. Radnor JM, Oliver JL, Waugh CM, Myer GD, Moore IS, Lloyd RS. The influence of growth and maturation on stretch-shortening cycle function in youth. Sports Medicine. 2018 Jan 1;48(1):57-71. https://link.springer.com/article/10.1007/s40279-017-0785-0

45. Hopkins, W.G., 2004. How to interpret changes in an athletic performance test [Online]. Sport Science.

46. Coutts, A. and Cormack, S., 2014. Monitoring the training response. High-performance training for sports, pp.71-84. 